\title{
Joint Queue Control and User Scheduling in MIMO Broadcast Channel under Zero-Forcing Multiplexing
}

\author{
Feng She, Hanwen Luo, Wen Chen and Xinbing Wang \\ Department of Electronic Engineering \\ Shanghai Jiaotong University \\ Shanghai 200030, P. R. China \\ Email: \{shefeng630;wenchen;xwang8\}@ sjtu.edu.cn
}

\begin{abstract}
This paper studies the problem of queue control and user scheduling in multi-antenna broadcast (downlink) systems under zero forcing beamforming (ZFBF) transmit strategy. In the system, we assume that the data packet arrives randomly to the buffered transmitter. By taking the broadcast channel as a controlled queueing system, we deduce the property of queue control function which maximizes the weighted system throughput while guarantees the delay fairness among users. We also present a low complexity user selection algorithm with the consideration of queue state and channel state together. Simulation results show that the joint queue control and user selection policy can achieve considerable fairness and stability among users.
\end{abstract}

\section{INTRODUCTION}

Multiple-input multiple-output (MIMO) system is well motivated for wireless communications through fading channels because of the potential improvement in transmit rate or diversity gain [1]. It is well known that multiple antennas can be easily deployed at base station in cellular systems. However, mobile terminals usually have a small number of antennas due to the size and cost constraint. Thus, it may appear that we can not obtain significant capacity benefit from the multiple transmit antennas. This is true with the transmit strategy of time division multiple access (TDMA) [2]. To solve the problem, multiuser must be served simultaneously. One way to accomplish this is called dirty paper coding (DPC), which is a multiuser encoding strategy based on interference presubtraction [3]. But DPC is of high complexity, and hence a much simple transmit strategy, zero forcing beamforming (ZFBF) technique has been proposed for space division multiple access (SDMA) to remove the cochannel interference in MIMO downlink systems [4]-[7].

In ZFBF multiplexing, an issue that has not been treated thoroughly in literature is how to control data queue in transmitter. This is the problem of resource allocation (such as power and bandwidth) and user scheduling in order to perform desirably with respect to the criteria such as fairness or throughput [9]. This problem has attracted great interest in the recent years [10]-[12].

Under random packet arrival, the notation of "fairness" is replaced by the notation of "stability" [9], [13], [14]. The system goal is to stabilize the $K$ transmission queues whenever the arrival rate is inside the stability region in the system.
User scheduling algorithms proposed in the previous literatures most commonly ignore queuing and randomness in packet arrivals and hence cannot offer stability guarantee. This is true in some scheduling algorithms that aim to satisfy a fairness criteria, such as proportional fair scheduling [2].

A guiding work which incorporate randomness and stability issues has been presented in [13], where the network capacity region is defined as the region of the input data rates making system stable. It is shown that this region is achievable by a maximum weight matching (weights related to queue length). Based on those definitions, [14] considers a broadcast scenario under time division and demonstrates a schedule algorithm that achieves the system capacity region. Similarly, [15] shows that a throughput optimal policy is a maximum weight matching in the form of $\max \sum_{i} \alpha_{i} q_{i} r_{i}$, where $q_{i}$ and $r_{i}$ are the queue length and service rate of the $i$ th user respectively, $\alpha_{i}$ is a nonnegative constant. Also in broadcast system, [16] compares several user scheduling policies.

In this paper we formulate the general broadcast scheduling problem where packet arrivals into the users queues are random processes. It is established that a throughput optimal schedule in the downlink channel is the one that chooses a group of users and corresponding rates from the dirty paper coding (DPC) region to maximize $g(q) \cdot r$, where $g(q)$ is a function of queue length $q$, and $r$ is transmit rate. However, this solution is of high complexity, not only due to the complexity of obtaining the DPC region, but also due to searching for an ordered subset of users out of total users. Therefore, ZFBF is an attractive in multiplexing technique scenario as it does not incur the user ordering problem and has lower coding complexity. Thus in this paper we consider ZFBF strategy which demonstrate good performance with low complexity. The contributions of this paper is as following

1) We give the property of the queue control function which can guarantee queue equal among the active users.

2) We have compared the fairness performance by using different queue control function.

3) We give the upper bound of the weighted throughput with queue control function.

4) We propose a low complexity user scheduling algorithm under ZFBF multiplexing. 


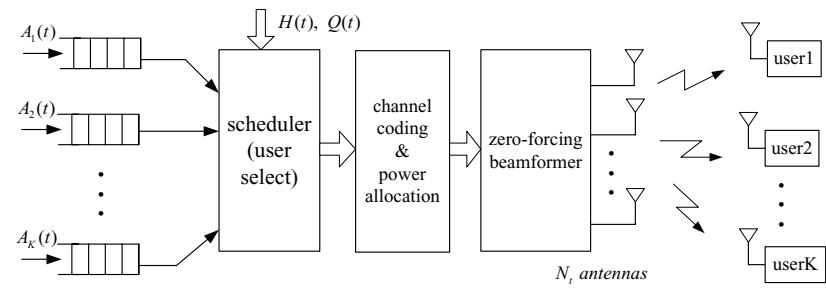

Fig. 1. Block diagram of multi-antenna broadcast system with queue control.

This paper is organized as follows. We outline the system model and the transmit strategy of ZFBF in section II. In section III, the queue and power control policy is presented. We analyze the scheduling algorithm under ZFBF multiplexing in section IV. The simulation results are presented in section V. Finally we conclude this paper in section VI.

Notation used in this paper are as follows: $(\cdot)^{T}$ denotes matrix transpose, $(\cdot)^{\mathbf{H}}$ denotes matrix conjugate-transpose, $E[\cdot]$ denotes statistical expectation, $(\cdot)^{\dagger}$ is pseudo inverse matrix and $\operatorname{tr}(\cdot)$ is matrix trace.

\section{System Model and Multiuser Transmit STRATEGIES}

\section{A. Multi-user broadcast channel model}

We consider a single cell MIMO BC system with a single base station supporting data traffic to $K$ users. As shown in Fig. 1, the base station is with $N_{t}$ transmit antennas and each of the user terminal has single receive antenna. We assume $K \geq N_{t}$. For simplicity, we assume that all the users are experience independent fading. Thus, the signal received by user $k$ is given by

$$
\boldsymbol{y}_{k}=\mathbf{h}_{k} \mathbf{x}+\mathbf{n}_{k}, \quad k=1, \ldots, K,
$$

where $\mathbf{x} \in \mathbb{C}^{N_{t} \times 1}$ is the transmit signal vector with a power constraint $\operatorname{tr}\left(E\left[\mathbf{x x}^{H}\right]\right) \leq P$, and $\mathbf{n}_{k}$ is complex Gaussian noise with unit variance per vector component (i.e., $E\left[\mathbf{n n}^{H}\right]=$ I). $\mathbf{h}_{k} \in \mathbb{C}^{1 \times N_{t}}$ is the multiple-input single-output (MISO) channel gain matrix to the $k$ th user.

At transmitter, we employ the ZFBF transmit strategy. In ZFBF, the scheduler first selects an active user set $S \subset$ $1,2, \ldots, K$, where $|S| \leq N_{t}$. Then, the transmitter assigns different beamforming direction to each data stream in such a way that the interference at each receiver is completely suppressed.

Denote $h_{i}, i=1, \ldots,|S|$ as the channel to the $i$ th active user, and define $H(S)=\left[h_{1}^{T}, \ldots, h_{|S|}^{T}\right]$ as the channel matrix of active user set. The transmit signal is expressed as

$$
\boldsymbol{x}=\sum_{i=1}^{|S|} \sqrt{P_{i}} w_{i} s_{i}
$$

where $s_{i}, w_{i}$, and $P_{i}$ are data symbol, beamforming vector, and transmit power for the $i$ th active user, respectively. Then the received signal at the $i$ th active user is given by

$$
\boldsymbol{y}_{i}=\sqrt{P_{i}} h_{i} w_{i} s_{i}+\sum_{j=1, j \neq i}^{|S|} \sqrt{P_{j}} h_{i} w_{j} s_{j}+n_{j},
$$

and the sum rate achieved by ZFBF is [4]

$$
R_{B F}=\max _{w_{k}, P_{k}} \log \left(\frac{1+\sum_{j=1}^{|S|} P_{j}\left|h_{k} w_{j}\right|^{2}}{1+\sum_{j=1, j \neq k}^{|S|} P_{j}\left|h_{k} w_{j}\right|^{2}}\right),
$$

subject to

$$
\sum_{k=1}^{K}\left\|w_{k}\right\|^{2} P_{k} \leq P .
$$

In this paper, we assume that the base station has perfect channel state information (CSI) of all the downlink channels, while each of the users only has the CSI of its own downlink channel and does not know the CSI of downlink channel of other users.

Fig. 1 also depicts a cellular network with $K$ flows transmitter buffers in the base station. In Fig. 1, $H(t), Q(t)$ and $A_{i}(t)$ are channel state information, queue state information and data arrival rate of user $i$ respectively. We assume that time is slotted, and let $q_{k}(t)$ denotes the size of the $k$ th queue at the beginning of the time slot $t, a_{k}(t)$ denotes the number of arrivals to queue $i$ in time slot $t$, and $r_{k}(t)$ denote the amount of service rate offered to queue $k$ in slot $t$. We assume that each of these parameters can only take nonnegative. Then the evolution of the size of the $k$ th queue is given by

$$
q_{k}(t+1)=\left(q_{k}(t)+a_{k}(t)-r_{k}(t)\right)^{+},
$$

where $(y)^{+}=\max \{y, 0\}$, and $k=1, \ldots, K$.

\section{B. Multi-antenna ZFBF}

In this subsection, zero forcing beamforming (ZFBF) in multi-antenna broadcast channel (BC) is introduced. It is well known that multiple transmit antennas can potentially yield an $N_{t}$-fold increase in the sum capacity, where $N_{t}$ is the number of transmit antennas. [2] shows that employing ZFBF to a set of $N_{t}$ nearly orthogonal users with large channel norms is asymptotically optimal as the number of users grows large.

In ZFBF, the beamforming matrix takes a pseudo inverse of the channel matrix. By this way, a respective data stream of each transmit antenna can be decoupled and thus sent to its desired user without interfering with other users. In multiuser MISO case, we first select a user subset $S$ to be served together, where subset size $|S| \leq N_{t}$, and then build up the corresponding channel matrix $H(S)$ of the user set. The beamforming matrix $\mathcal{W}(S)$ is written as

$$
\mathcal{W}(S)=H(S)^{H}\left(H(S) H(S)^{H}\right)^{-1} .
$$

As a result, the achievable throughput of ZFBF for a given user set $S$ is given by

$$
R_{Z F B F}(S)=\max _{\sum_{i \in S} b_{i}^{-1} P_{i} \leq P} \sum_{i \in S} \log _{2}\left(1+P_{i}\right),
$$


where

$$
b_{i}=\frac{1}{\left[\left(H(S) H(S)^{H}\right)^{-1}\right]_{i, i}},
$$

is the effective channel gain of the $i$ th user, and the power $P_{i}$ is obtained by waterfilling.

Note that the $b_{i}$ in (9) has an important geometric interpretation as noted in [4], i.e., the distance squared of the $i$ th user channel from the span of every other user channels in the activation set.

Thus, (??) gives us sense to define a queue function $g(q)$ to guarantee the equal size for the active user subset.

\section{Joint Scheduling Policy of ZFBF Multiplexing}

In this section, we first analysis the upper bound of weighted throughput with queue function, and then present a low complexity user scheduling algorithm.

\section{A. Upper bound of weighted throughput}

Define $f(q, r)=\sum_{i \in S} g\left(q_{i}\right) r_{i}$, our goal is to

$$
\max f(q, r)=\max \sum_{i \in S} g\left(q_{i}\right) r_{i},
$$

where $S$ is the active user set. Similar to the analysis in [17], we can get the following theorem.

Theorem 1: With a total power constraint, we have

$$
\begin{aligned}
& \max f(q, r)=\max \sum_{i \in S} g\left(q_{i}\right) r_{i} \\
& \leq \max \left(\sum_{i \in S} g\left(q_{i}\right)\right)\left(\log _{2}\left(1+\frac{P}{\operatorname{tr}\left(W_{S}^{-1}\right)}\right)\right. \\
&\left.+D\left(g\left(q_{S}\right) \| b_{S}\right)\right) .
\end{aligned}
$$

where $W_{S}=H(S) H(S)^{H}, b_{i}=\frac{1}{\left(W_{S}^{-1}\right)_{i, i}}, \quad \sum_{i \in S} P_{i} / b_{i} \leq P$, $g\left(q_{S i}\right)=\frac{g\left(q_{i}\right)}{\sum_{i \in S} g\left(q_{i}\right)}, b_{S i}=\frac{b_{i}}{\sum_{i \in S} b_{i}}$, and $D(\cdot \| \cdot)$ denotes the Kullback Leibler distance.

\section{B. Selecting semi-orthogonal user set with waterfilling}

For a given set of channel gains and corresponding queue length, the weighted sum rate is maximized by an orthogonal user set. As mentioned above, such a set of user has the largest conditional "geometry gain". In addition, since the channel matrix is orthogonal, zero-forcing incurs no power penalty for inverting the channel and thus can approach the dirty-paper coding rate region [2].

While in practice, for finite user number $K$, the probability of existence of an orthogonal set is zero. Thus, we consider "nearly" orthogonal user sets. To be precise, define two vectors $v_{1}$ and $v_{2}$ to be $\alpha$-orthogonal if

$$
\frac{\left|v_{1} v_{2}^{H}\right|}{\left\|v_{1}\right\| \cdot\left\|v_{2}\right\|} \leq \alpha
$$

Here, we construct a low complexity user group by combing the semi-orthogonal user selection and waterfilling (SUSWF) algorithm, as outlined next.
1) Initialization:

$$
S_{1}=\{1, \ldots, K\} ; i=1 ; S_{0}=\phi \text { (empty set). }
$$

2) For each user $k \in S_{i}$, calculate $g_{k}$, the component of $H_{k}$ orthogonal to the space spanned by $\left\{g_{1}, \ldots, g_{i-1}\right\}$

$$
g_{k}=H_{k}-\sum_{j=1}^{i-1} \frac{H_{k} g_{j}^{H}}{\left\|g_{j}\right\|^{2}} g_{j}
$$

When $i=1$, let $g_{k}=H_{k}$.

3) Select the $i$ th user as follows:

$$
\begin{gathered}
s_{i}=\arg \max g\left(q_{k}\right) \log _{2}\left(1+\frac{P}{N_{t}}\left\|g_{k}\right\|^{2}\right) \\
S_{0}=S_{0} \cup\left\{s_{i}\right\}, \quad h_{i}=h_{s_{i}}, \quad g_{i}=g_{s_{i}}
\end{gathered}
$$

4) Do Waterfilling among selected users and reject the users which are under the waterlevel in this step [18].

5) If $\left|S_{0}\right|<N_{t}$, then calculate $S_{i+1}$, which is the set of unselected users which is $\alpha$-semiorthogonal to $g_{i}$

$$
S_{n+1}=\left\{k \in S_{n}, k \neq s_{i} \mid \frac{\left|h_{k} g_{i}^{H}\right|}{\left\|h_{k}\right\|\left\|g_{i}\right\|}<\alpha\right\}
$$

5) Increase $i$ by 1 .

where $\alpha$ is a small positive constant which is defined as (12). If $S_{n+1}$ is nonempty and $\left|S_{0}\right|<N_{t}$, then go to step 2 . Otherwise, the algorithm is finished.

User scheduling algorithm based on semi-orthogonality has been proposed in [19] and [20]. A similar algorithm to the $S U S$ has been proposed in [2]. While in [2], stability and queue control are not considered. Furthermore, we added waterfilling process in user selection. [18] point out that in the algorithm of user selection, the water level will decrease in the next iteration. Thus the users rejected in this iteration will be rejected in the next iteration. Therefore, the advantage of step 4 is that it can reduce the complexity (running time) by eliminating those weaker users in this subsequent iteration.

\section{Simulation Result And Discussion}

In this section, numerical results for the queue control of weighted SUSWF are presented. The considered multiuser MIMO system in broadcast channel with the number of transmit antenna is $N_{t}=4$, and each user has single receive antenna. We assume that the power in the simulation is $P=15 \mathrm{~dB}$. With spatial multiplexing, the number of data streams does not exceed the number of transmit antennas. We also assume that the channels between different transmit and receive antennas are independent.

To be simple, we define a special queue function $g(q)=q^{m}$, and consider 20 users in simulation. We assume that at least one user queues are served in one time slot. In this scenario, the scheduling algorithm is equivalent to serving the users that solve $f(t)=\arg \max _{i \in S} \sum q^{m}(t) r(t)$. We consider each queue with mutually independent arrival processes such that $A_{k}(t)$ is with rate $\lambda_{k}$ (bits/channel use), and let the rate vectors, $\left(\lambda_{1}(t), \ldots, \lambda_{K}(t)\right)$ be randomly created between $[0,1]$. We 


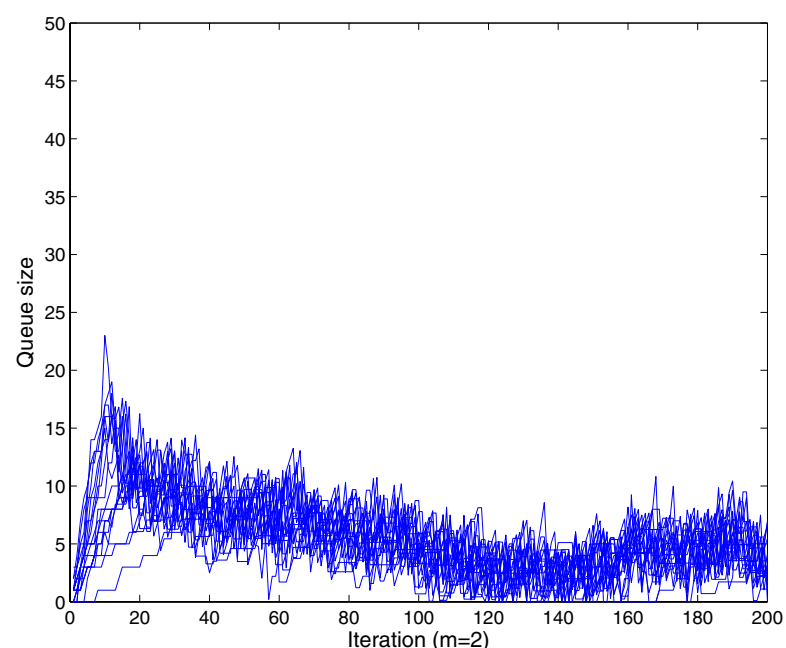

Fig. 2. The queue length in multi-antenna broadcast channel with $m=2$.

also assume that the number of arrivals in each slot to each queue is Poisson distributed.

A. Experiment 1: The first experiment tests the average queue length with $m=2$ in queue control function $q^{m}$. In this experiment, the queue control carry out from the 15 th iteration. Fig. 2 shows that by queue controlling function, the system can reach stable after few times of iteration.

B. Experiment 2: This experiment is about the fairness among multi-users. Here, we use the variance value of the multi-user queue length to denote the fairness. In other words, large variance value implies poor fairness while small variance value denotes good fairness performance among users. In this experiment, we calculate the normalized variance value with different $m$. Fig. 3 shows that the variance decrease with the increment of $m$. Thus, to get the fairness among users, the selected $m$ should not be very small.

C. Experiment 3: The third experiment is about the average sum throughput of different $m$. Due to the variance value is very large when $m<1$, which is shown in the above experiment, we only consider the scenario of $m \geq 1$ in this experiment. By 10000 times of iteration, Fig. 4 shows that the average throughput is almost the same, which implies that $m \geq 1$ is a good option.

D. Experiment 4: This experiment tests the average queue length with different $m$. We only consider $m \geq 1$ as the above experiment. We calculate the average queue size by 10000 times of iteration. Fig. 5 shows that the sum queue length increases slightly with the increment of $m$.

D. Experiment 5: The last experiment is about the stability of the system with queue control. In this experiment, we test the input rate and the output rate, and we also test the queue size of each user. We carry queue control from the 20th iteration. From Fig. 6, we can see that the system with queue control is stable, and the input rate and output rate also show the equilibrium.

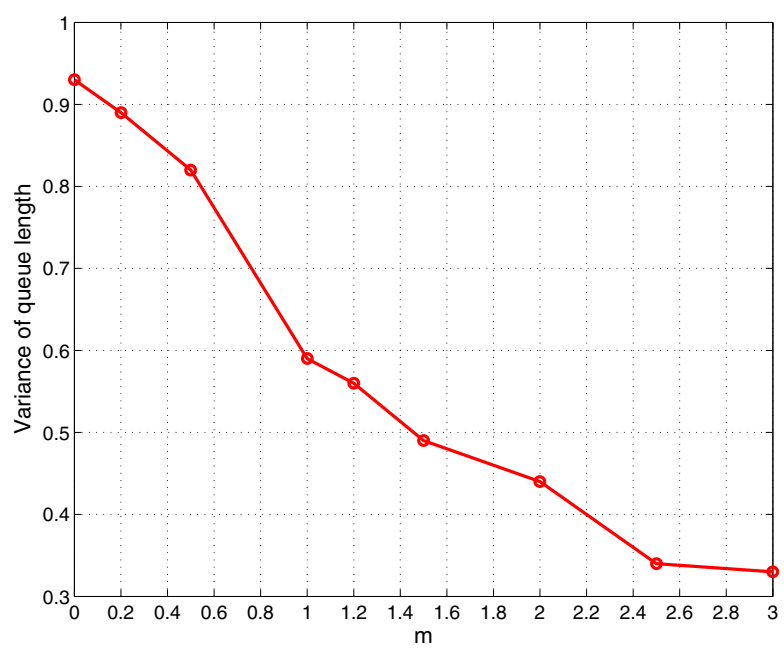

Fig. 3. Variance of queue length with different $m$.

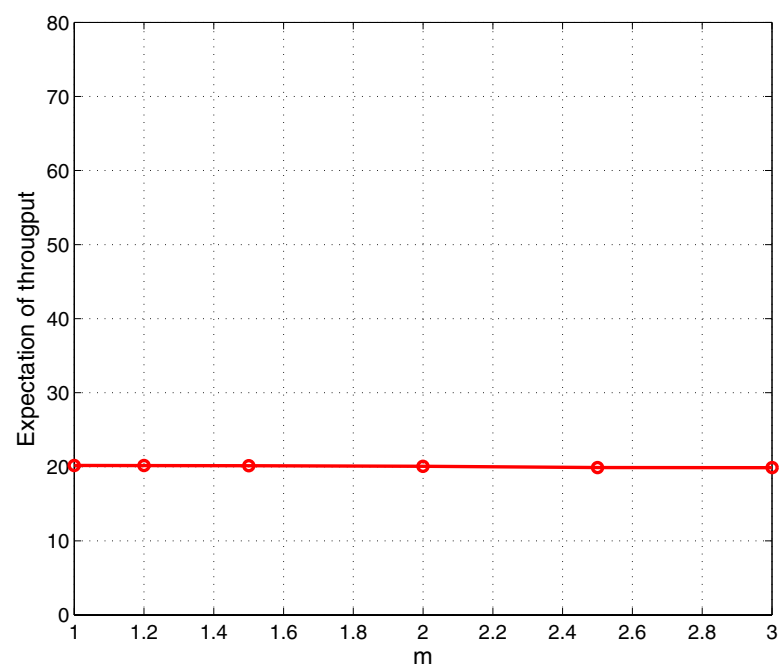

Fig. 4. Expectation of throughput with different $\mathrm{m}$.

\section{CONCLUSION}

In this paper, we have presented a frame work of joint queue control and user scheduling. We point out that to reach queue length fairness (equal queue length in transmitter buffer) among multi-users. The function of queue length should be correctly selected. Based on the queue length function, we derive the upper bound of the weighted throughput. By jointly consider channel gains and queue states, we present a low complexity user selection algorithm which is named SUSWF.

In simulation experiments, we select a special queue control function as $g(q)=q^{m}$ in simulation, and the simulation results show that queue control policy is critical in fairness user scheduling. In this paper, we take a special case of queue control function, while the design of the queue function is still very challenge and interesting future works. 


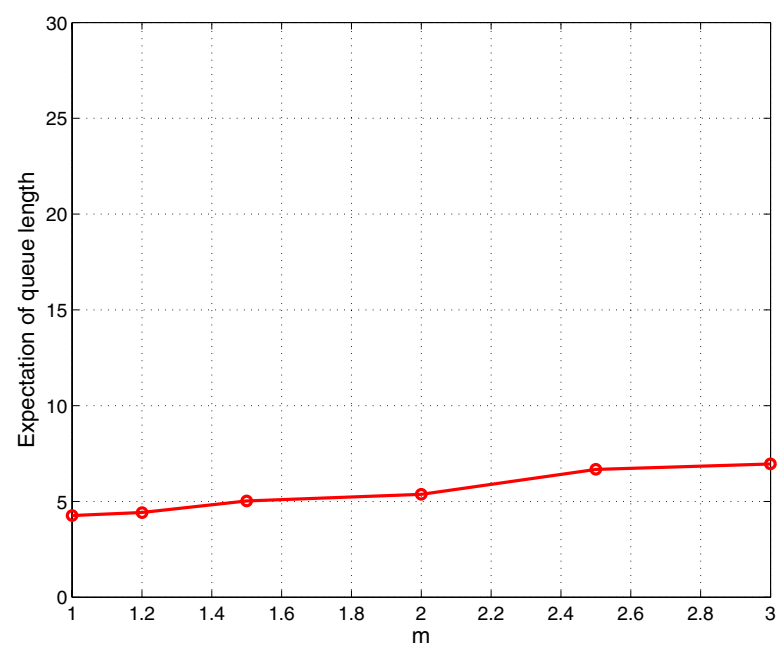

Fig. 5. The expectation of queue length with different $m$.
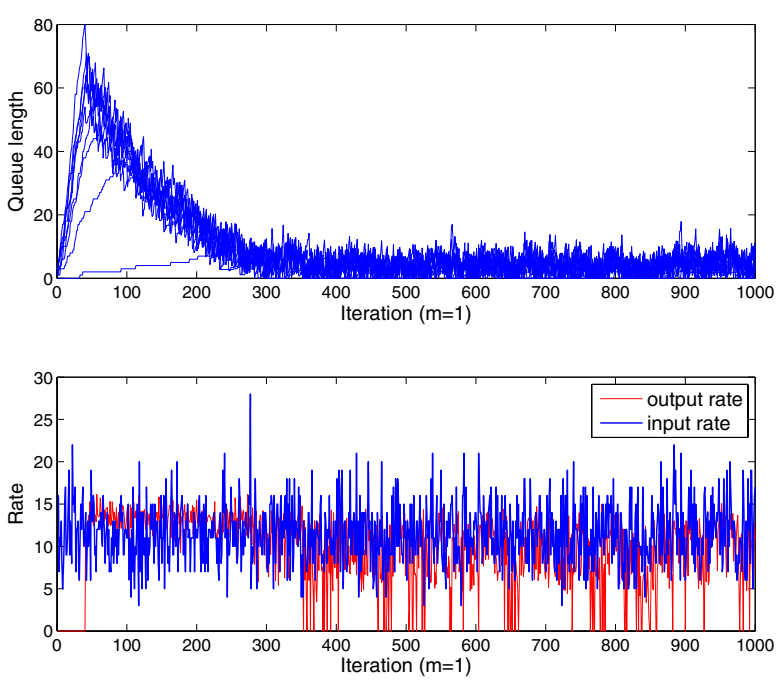

Fig. 6. The input-output throughput and queue length with $m=1$.

\section{ACKNOWLEDGMENT}

The authors would like to thank to Da Mao Yang and Hong Xing Li for their helpful discussion.

This work is supported by NSF China \#60572157, \#60672067, \#60702046 by NSF Shanghai \#06ZR14041, by Shanghai-Canada NRC \#06SN07112, by Cultivation Fund of the Key Scientific and Technical Innovation Project, Ministry of Education of China \#706022, by Program for New Century Excellent Talents in University \#NCET-06-0386, and by Pujiang Talent\#07pj4046.

\section{REFERENCES}

[1] S. M. Alamouti, "A simple diversity techniques for wireless communications," IEEE J. Select. Areas commun., vol. 16, pp.1451-1458, Oct. 1998.
[2] T. Yoo, A. Goldsmith, "On the optimality of multi-antenna broadcast scheduling using zero-forcing beamforming," IEEE J. Sel. Areas Commun., vol. 24, no. 3, pp. 528-541. May. 2006.

[3] M. H. M. Costa, "Writing on dirty paper," IEEE trans. Inf.Theory, vol. 29, no. 3, pp. 439-441. Mar. 1983.

[4] G. Caire, S. Shamai, "On the achievable throughput of a multiantenna Gaussian broadcast channel," IEEE trans. Inf.Theory., vol. 49, no. 7, pp. 1691-1706. Jul. 2003.

[5] N. Jindal, A. Goldsmith, "Dirty-paper coding versus TDMA for MIMO broadcast channels," IEEE trans. Inf.Theory, vol. 51, no. 5, pp. 17831794. May. 2005.

[6] Q.H. Spencer, A.L. Swindlehurst and M. Haardt, "Zero-forcing methods for downlink spatial multiplexing in multiuser MIMO channels," IEEE Trans. Signal Processing, vol. 52, no.2 pp.461-471, Oct. 2004.

[7] Q.H. Spencer, C.B. Peel, A.L. Swindlehurst and M. Haardt, "An introduction to the multi-user MIMO downlink," IEEE Communications Magazine, vol. 42, no. 10 pp.60-67, Oct. 2004.

[8] M. J. Neely, E. Modiano and C. E. Rohrs, "Power allocation and Routing in multibeam Satellites with time-varying channels," IEEE/ACM Transaction on Networking, vol. 11, pp. 138-152, Feb. 2003.

[9] E. M. Yeh, A. S. Cohen, "information theory, queueing, and resource allocation in multi-user fading communications," in Proc. of the 2004 CISS, Princeton, NJ, Mar. 2004.

[10] M. Andrews, K. Kumaran, K. Ramannan, A. Stolyar, and P. Whiting, "Prociding quality of service over a shared wireless link," IEEE Communications Magazine, vol. 39, no.2 pp.150-154, Feb. 2001.

[11] P. Viswanath, D. Tse, and R. Laroia, "Opportunistic beamforming using dumb antennas," IEEE trans. Inf.Theory, vol. 48, no. 6, pp. 1277-1294. May. 2002.

[12] H. Weingarten, Y. Steinberg, and S. Shamai, "The capacity region of the Gaussian multiple-input multiple-output broadcast channel," IEEE trans. Inf.Theory, vol. 52, no. 9, pp. 3936-3964. Sep. 2006.

[13] L. Tassiulas and A. Ephremides, "Stability properties of constrained queueing systems and scheduling policies for maximum throughput in multihop radio networks," IEEE trans. Automat. Contr., vol. 37, no. 12, pp. 1936-1948. Dec. 1992.

[14] M. Neely, E. Modiano, and C. Rohrs,, "Power allocation and routing in multibeam satellites with time-varying channels," IEEE/ACM trans. Networking., vol. 11, no. 1, pp. 138-152. Feb. 2003.

[15] H. Vishwanathan and K. Kumaran, "Rate scheduling in multiple antenna downlink wireless systems," IEEE trans. Commun., vol. 53, no. 4, pp. 645-655. Apr. 2005.

[16] M. Airy, S. Shakkottai, and J. R.W. Heath, "Limiting queuing models for scheduling in multi-user mimo wireless systems," in Proc. of the 2nd IASTED Conf. on Communications, Internet \& Info Technology, Scottsdale, Arizona, Nov. 2003.

[17] C. Swannack, E. Uysal-Biyikoglu, and G. W. Wornell, "Low complexity scheduling for maximizing throughput in the MIMO broadcast channel," in Proc. 42nd Annual Allerton Conf. on Communicaton, Control, and Computing, Monticello, Illinois, 2004.

[18] G. Dimić, N. D. Sidiropoulos, "On downlink beamforming with greedy user selection: Performance analysis and a simple new algorithm," IEEE trans. Signal.Processing, vol. 53, no. 10, pp. 3857-3868. Oct. 2005.

[19] Z. Tu and R. Blum, "Multiuser diversity for a dirty paper approach," IEEE Commun. Lett., vol. 7, no. 8, pp. 370-372, Aug. 2003.

[20] R. Zhang, Y. C. Liang, and J. Cioffi, "Throughput comparision of wireless downlink transmission schemes with multiple antennas," in Proc. IEEE Int. Conf. Commun., 2005. 